\title{
Evaluation of diagnostic tests for Wuchereria bancrofti infection in Brazilian schoolchildren
}

\author{
Paula Oliveira ${ }^{[1]}$, Cynthia Braga ${ }^{[2]}$, Neal Alexander ${ }^{[3]}$, Eduardo Brandão ${ }^{[1]}$, \\ Almerice Silva ${ }^{[2]}$, Leandro Wanderley ${ }^{[2]}$, Ana Maria Aguiar ${ }^{[1]}$, George Diniz ${ }^{[4]}$, \\ Zulma Medeiros ${ }^{[1]}$ and Abraham Rocha ${ }^{[1]}$
}

[1]. Serviço de Referência Nacional em Filarioses, Centro de Pesquisas Aggeu Magalhães, Fundação Oswaldo Cruz, Recife, PE. [2]. Departamento de Parasitologia, Centro de Pesquisas Aggeu Magalhães, Fundação Oswaldo Cruz, Recife, PE. [3]. Department of Infectious Disease Epidemiology, London School of Hygiene and Tropical Medicine, London, UK. [4]. Departamento de Saúde Coletiva, Centro de Pesquisas Aggeu Magalhães, Fundação Oswaldo Cruz, Recife, PE.

\begin{abstract}
Introduction: Since the launch of the Global Programme to Eliminate Lymphatic Filariasis, more than $70 \%$ of the endemic countries have implemented mass drug administration (MDA) to interrupt disease transmission. The monitoring of filarial infection in sentinel populations, particularly schoolchildren, is recommended to assess the impact of MDA. A key issue is choosing the appropriate tools for these initial assessments (to define the best intervention) and for monitoring transmission. Methods: This study compared the preMDA performance of five diagnostic methods, namely, thick film test, Knott's technique, filtration, Og4C3-ELISA, and the AD12-ICT card test, in schoolchildren from Brazil. Venous and capillary blood samples were collected between $11 \mathrm{pm}$ and $1 \mathrm{am}$. The microfilarial loads were analyzed with a negative binomial regression, and the prevalence and associated $95 \%$ confidence intervals were estimated for all methods. The accuracies of the AD12-ICT card and Og4C3-ELISA tests were assessed against the combination of parasitological test results. Results: A total of 805 schoolchildren were examined. The overall and stratified prevalence by age group and gender detected by Og4C3-ELISA and AD12-ICT were markedly higher than the prevalence estimated by the parasitological methods. The sensitivity of the AD12-ICT card and Og4C3-ELISA tests was approximately $100 \%$, and the positive likelihood ratios were above 6. The specificity of the Og4C3-ELISA was higher than that of the AD12-ICT at different prevalence levels. Conclusions: The ICT card test should be the recommended tool for monitoring school-age populations living in areas with ongoing or completed MDA.
\end{abstract}

Keywords: Filariasis diagnosis. ELISA. Membrane filtration. Laboratory tests.

\section{INTRODUCTION}

The Global Programme to Eliminate Lymphatic Filariasis (GPELF), launched by the World Health Organization (WHO) in 1997, has targeted the elimination of the disease as a public health problem by 2020 . Of the 73 endemic countries, 56 have implemented mass drug administration (MDA) programs to interrupt disease transmission. By 2012, more than 4.4 billion doses of antifilarial drugs had been delivered to almost 984 million people worldwide ${ }^{1}$.

Effective monitoring and evaluation of the impact of MDA programs are important for achieving the goal of disease elimination. A number of diagnostic tools designed to assess filarial infection are standardized and currently recommended by the $\mathrm{WHO}^{1}$. These tools include both parasitological methods for detecting microfilaremia and tests for detecting

Address to: Dr ${ }^{\mathrm{a}}$ Paula Oliveira. Serviço de Referência Nacional em Filarioses/ Dept $^{\circ}$ Parasitologia/CPqAM. Av. Prof. Moraes Rego, s/n, Campus da UFPE, Cidade Universitária, 50670-420 Recife, PE, Brasil.

Phone: 5581 2101-2579; Fax: 5581 2101-2671

e-mail: brandaoec@gmail.com; paula.oliveira@cpqam.fiocruz.br

Received 6 May 2014

Accepted 30 June 2014 the circulating antigens of Wuchereria bancrofti, such as the immunochromatographic test (AD12-ICT card test) and enzyme-linked immunosorbent assay (Og4C3-ELISA) ${ }^{1}$. The choice of which diagnostic tools to use is usually driven by characteristics such as accuracy (sensitivity and specificity), feasibility of use in the field, technical skills required, and $\operatorname{cost}^{1}$. It is critical to define the most appropriate diagnostic tools for the initial assessments of lymphatic filariasis (LF) infection in the population (to define the best intervention), as well as to monitor transmission in areas undergoing MDA, because the performance of such tools can vary substantially according to the epidemiologic and demographic characteristics of the study population ${ }^{2,3}$.

The WHO has recommended the monitoring of filarial infection in sentinel populations, particularly schoolchildren ${ }^{4-6}$, due to children's lower duration of exposure (many of these children were born following or during the start of the intervention), which allows for a more accurate assessment of transmission ${ }^{5}$. However, the pediatric population usually exhibits lower microfilarial loads and higher proportions of amicrofilaremic and asymptomatic infections compared with adults ${ }^{7-9}$. As these features will most likely influence the operating characteristics of diagnostic tests, it is important to evaluate them in children. This study evaluated the preMDA performance of several diagnostic methods, namely, the thick film test, Knott's concentration method, filtration, 
Og4C3-ELISA, and the AD12-ICT card test, in children from an endemic area of Brazil prior to the initiation of an MDA program.

\section{METHODS}

\section{Study setting}

The study was conducted in the three neighborhoods of the City of Olinda, Metropolitan Region of State Pernambuco, Brazil, with the highest prevalence of microfilaremia, according to a survey conducted in 1998 (unpublished data), between 2007 and 2010 . The city has an area of $41,659 \mathrm{~km}^{2}$ and a population of 377,779 , as reported in the last census ${ }^{10}$, and is considered one of the remaining foci of filariasis in Brazil ${ }^{11,12}$.

Children between the ages of 4 and 15 years who attended five public schools in these neighborhoods (Alto da Conquista, Alto da Bondade, and Sapucaia) participated in this study.

\section{Data collection}

Parents or guardians were informed of the main study objectives, and consent was sought for the children's participation. After obtaining written consent, each child's data (name, address, age, and gender) were obtained using a standardized form. The blood samples were obtained between 23:00 and 1:00. Initially, capillary blood samples were obtained by finger prick to perform the thick blood film $(\sim 60 \mu \mathrm{L}$ of blood $)$ and the AD12-ICT card $(100 \mu \mathrm{L})$ tests. A venous blood sample $(7 \mathrm{~mL})$ was then collected to perform the filtration, Knott's concentration, and $\mathrm{Og} 4 \mathrm{C} 3$-ELISA tests. This sample was partitioned into tubes containing ethylenediamine tetraacetic acid (EDTA), tubes containing $2 \%$ formalin, and dry tubes to perform the filtration, Knott's concentration test, and $\mathrm{Og} 4 \mathrm{C} 3$ ELISA test, respectively. The blood samples were transported in a refrigerated container to the laboratory, stored at $2-8^{\circ} \mathrm{C}$, and processed on the following day.

\section{Laboratory procedures}

I) Parasitological methods: A) Thick blood film (TBF) test: The smear was left to dry overnight at room temperature. The slides were then dehemoglobinized in water, fixed in methanol for $3 \mathrm{~min}$, stained according to previously described procedures, and read with an optical microscope ${ }^{13}$. B) Knott's concentration method: Venous blood $(\sim 1 \mathrm{~mL})$ was drawn into a tube containing $9 \mathrm{~mL}$ of $2 \%$ formalin and processed according to the methodology described by Knott ${ }^{14}$. C) Filtration technique: Blood $(\sim 1 \mathrm{~mL})$ was filtered through a polycarbonate membrane with a width of $13 \mathrm{~mm}$ and pore size of $3 \mu \mathrm{m}$, as described by Dennis and Kaen ${ }^{15}$. These three parasitological tests rely on the microscopic visualization of microfilariae. Thus, their specificity, although not their sensitivity, can be considered to be very high. II) Immunological methods: A) AD12-ICT card test: This test detects circulating filarial antigen (CFA) using the monoclonal antibody AD12, which recognizes a 200-kDa filarial antigen from either adult worms or microfilariae ${ }^{16}$. The test was performed according to the manufacturer's instructions and read by trained technicians in the field after $10 \mathrm{~min}$. The visualization of the two lines (test and control) was interpreted as a positive result. B) $O g 4 C 3$-ELISA: The test was performed as recommended by the manufacturer (TropBio ${ }^{\circledR}$, Townsville, Australia). Samples with an antigen concentration $\geq 128 \mathrm{~mL}$ units (UA) were interpreted as positive ${ }^{17}$. This test detects CFA using a monoclonal antibody against antigens of the bovine parasite Onchocerca gibsoni, which has no known cross-reactivity with other human helminthes. The test provides quantitative results that enable the observation of variations in antigenemia levels after treatment ${ }^{18-24}$.

\section{Data analysis}

The reading and interpretation of all laboratory tests were performed by observers who were blinded to the other test results. Data entry and analysis were performed using EpiInfo and R software. Mean microfilarial densities were estimated and compared with a negative binomial regression ${ }^{25}$. The parasite load, determined by the three parasitological techniques (thick smear, Knott's concentration, and membrane filtration), and antigenemia, determined by the $\mathrm{Og} 4 \mathrm{C} 3$-ELISA in terms of unit antigens (UA), were stratified by age group and gender. Antigenemia was compared between groups with a KruskalWallis test. The mean antigenemia was quoted for descriptive purposes only, as most subjects were negative and, therefore, the median values were zero.

The infection prevalence identified by each technique was stratified by age group and gender. Statistical comparisons between groups were made using chi-square $\left(\chi^{2}\right)$ and KruskalWallis tests for qualitative and continuous results, respectively. A p-value of 0.05 was considered statistically significant.

The sensitivity, positive predictive value (PPV), negative predictive value (NPV), positive likelihood ratio (LR+), negative likelihood ratio (LR-), and their respective 95\% confidence intervals (CIs) for the AD12-ICT card test and Og4C3-ELISA were estimated against the gold standard of the combination of the parasitological techniques (thick blood film, Knott's concentration, and filtration). The LR + measures how many times more likely it is that a positive result is obtained for individuals with the disease than for individuals without the disease, whereas the LR- reveals how many times more likely it is that a negative result is obtained for individuals with the disease than for individuals without the disease. Values of LR+ above 10 and LR- below 0.1 provide strong evidence to confirm or rule out the diagnosis of disease, respectively ${ }^{26}$.

The parasitological tests are assumed to have $100 \%$ specificity but are of unknown sensitivity, as many individuals can harbor adult worms without demonstrating microfilaremia ${ }^{12}$. Consequently, the sensitivity of the ICT card test and the $\mathrm{Og} 4 \mathrm{C} 3$ ELISA can be estimated, but not their specificity. Because the specificity cannot be directly evaluated, we estimated the specificities of the Og4C3-ELISA and AD12-ICT card tests as a function of the prevalence of filariasis using the equation derived by Staquet et al. ${ }^{27}$. This calculation allows for the comparison of the specificities of the Og4C3-ELISA and AD12-ICT card tests for different prevalences ${ }^{27}$ of samples with viable worms (adult or microfilaria) or circulating antigen. 
The performances of the AD12-ICT card and thick film tests were compared according to sections 2.2 and 3.2.1 of Hayen et al. ${ }^{28}$, with the filtration test used as the reference standard. There are eight possible combinations of the three tests, but we considered only the four cells in which the filtration test is positive. Due to the high specificity of filtration, these cells can reliably be considered to be truly positive. The Table 1 was adapted from Table 2 of Hayen et al. ${ }^{28}$. The true positive fractions in the AD12-ICT card and thick blood film tests can be estimated as $(r+t) / n_{+}$and $(r+s) / n_{+}$, respectively, and the McNemar test can be used to assess the difference between these values.

\section{Ethical considerations}

This study was approved by the Ethics Committee of the Aggeu Magalhães Research Center (CAEE: 0069.0.095.000-06). All of the participants with a positive result in any of the tests were treated and underwent clinical and laboratory examinations.

TABLE 1 - Contingency table of two tests in a paired design among those positive for the reference standard (filtration). Test $\mathrm{N}$ is a 'new' test, e.g., the AD12-ICT card test, and test $\mathrm{E}$ is an existing test, e.g., the thick blood film test. Adapted from Table 2 of Hayen et al. ${ }^{28}$.

\begin{tabular}{lccc}
\hline & \multicolumn{3}{c}{ Test N } \\
\cline { 2 - 4 } & positive & negative & total \\
\hline Test E & $r$ & $s$ & $r+s$ \\
positive & $t$ & $u$ & $t+u$ \\
negative & $r+t$ & $s+u$ & $n_{+}$
\end{tabular}

AD12-ICT: immunochromatographic test using the monoclonal antibody AD12. Note: $\boldsymbol{n}_{+}=\boldsymbol{r}+\boldsymbol{s}+\boldsymbol{t}+\boldsymbol{u}$, where $\boldsymbol{n}_{+}$: number of people with the disease; $\boldsymbol{r}$ : true positive; $\boldsymbol{s}$ : false negative; $\boldsymbol{t}$ : false positive, and $\boldsymbol{u}$ : true negative.

\section{RESULTS}

A total of 805 schoolchildren participated in the study and agreed to be tested via finger prick. Overall, 783 children were tested by the thick blood film test and 797 by the AD12-ICT card test. Consent for taking venous blood samples was obtained from only 554 participants, all of whom were examined by Knott's concentration method, 546 by the filtration technique, and 545 by the Og4C3-ELISA test. In 487 schoolchildren, filarial infection was assessed with all laboratory tests.

The microfilaria (MF) loads ranged from 0 to $216 \mathrm{MF} / 60 \mu \mathrm{L}$, 0 to $1,423 \mathrm{MF} / \mathrm{mL}$, and 0 to $1,834 \mathrm{MF} / \mathrm{mL}$ according to the thick blood film, Knott's concentration, and filtration tests, respectively. No statistically significant variation in MF load was observed between age groups or genders when assessed by any of these three methods (Table 2). The mean values of filarial antigenemia also did not differ according to age group or gender (Table 2).

The estimated prevalence of filarial infection according to both filarial antigen tests (overall and stratified by age group and gender) was similar and markedly higher than the prevalence estimated by parasitological methods. This difference in estimates was more pronounced in the younger age group (4-9 years), in whom the prevalence of filarial infection estimated by the ICT and Og4C3-ELISA tests was approximately five times higher than that obtained by parasitological methods (thick blood film, Knott's concentration, or filtration test) (Table 3).

The MF prevalence estimated by either Knott's (4.6\%; 95\%CI: 3.1-6.8) or the filtration $(5.2 \%$; $95 \%$ CI: $3.5-$ $7.5 \%)$ method was slightly higher than the prevalence estimated by the thick film method. The prevalence of filariasis according to the parasitological methods was significantly higher in the 10-15-year-old age group than in the 4-9-year-old age group, whereas no difference between age groups was found by the immunological methods (AD12-ICT card test and Og4C3ELISA) (Table 3). The prevalence of filarial infection was higher in boys than in girls according to all techniques, although a statistically significant difference in the prevalence between the groups was observed only with the AD12-ICT card test (Table 3).

The comparative analysis of the performance of the AD12ICT card test and $\mathrm{Og} 4 \mathrm{C} 3$-ELISA against the gold standard (the combination of all parasitological test results) showed that both immunological tests exhibited $100 \%$ sensitivity. The PPVs were approximately $30 \%$, and the NPVs were close to $100 \%$. The $\mathrm{LR}+$ was 6.89 and 7.89 for the AD12-ICT card test and $\mathrm{Og} 4 \mathrm{C} 3-$ ELISA, respectively. Both LR- values were zero (Table 4).

Figure 1 shows the curves comparing the specificities of the AD12-ICT and Og4C3-ELISA tests over the feasible range of values of infection prevalence ( $5 \%$ to $20 \%$ ) based on the method of Staquet et al. Although the exact values are unknown, we can conclude from these ranges that a) both tests have relatively high specificities (84.4\% [95\% CI: 80.8-87.4] for the AD12ICT and 86.5\% [CI 95\%: 83.0-89.3] for the Og4C3-ELISA), and b) the Og4C3-ELISA has a slightly higher specificity than the AD12-ICT test.

The true positive fractions (TPFs) of the AD12-ICT card and thick blood film tests, calculated with the Hayen formula, were $100 \%(27+0 / 27)$ and $85.2 \%(23+4 / 27)$, respectively. This difference of $14.8 \%$ between the TPF values was statistically significant, suggesting that for each 1,000 schoolchildren with the infection, the AD12-ICT card test was able to detect 148 more cases than the thick blood film test. The TPFs for the AD12-ICT card test and Og4C3-ELISA were both $100 \%$. Accordingly, no difference could be found in the ability of the tests to detect cases of filarial infection. 


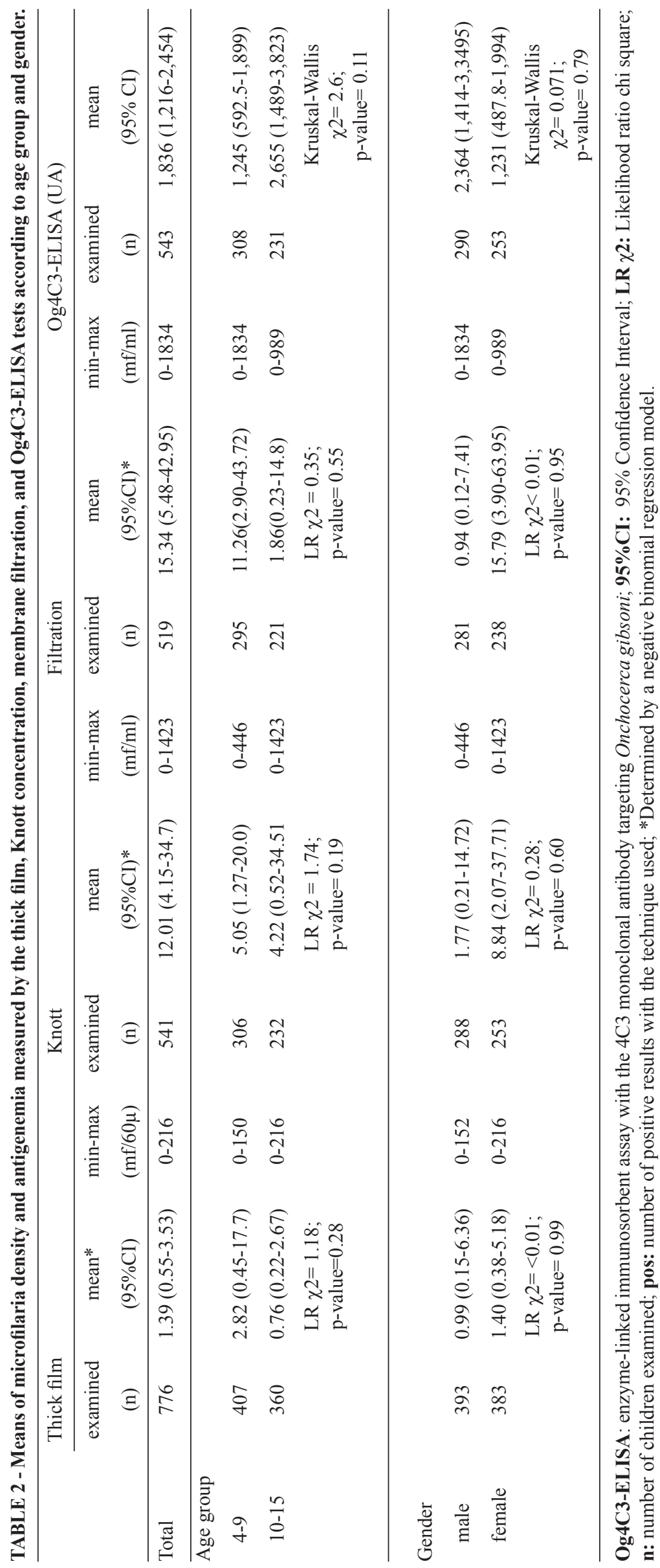

\section{DISCUSSION}

This study assessed the accuracy of tests in detecting bancroftian filariasis in a large and representative sample of schoolchildren from an endemic area in northeastern Brazil before the initiation of MDA. The performance evaluation of these tests in children is critical to evaluate the impact of the clinical and epidemiological characteristics of this population that may influence test performance ${ }^{2,3}$. This evaluation is of particular importance, as this demographic group has been recommended as a sentinel population by the WHO in whom to assess MDA effectiveness in areas undergoing this intervention.

The prevalence of filariasis estimated by the filarial antigen tests (AD12-ICT card test and Og4C3-ELISA) was far higher than that estimated by parasitological methods. The difference between the results of these methods was as much as fivefold in the group aged below 10 years. The comparative evaluation of the performance of the thick film test in relation to the AD12-ICT card test using the Hayen et al. ${ }^{28}$ formula also showed that in each group of 1,000 schoolchildren with filarial infection, the AD12-ICT card test was able to detect approximately 150 more cases than the thick film test. These results are in agreement with previous studies conducted in the school-age population ${ }^{29-39}$ and reinforce the usefulness of the AD12-ICT card test in screening for filarial infection in children for the assessment of MDA efficacy ${ }^{6,40}$. The greater sensitivity of the antigen tests can be explained by their ability to detect low-level microfilaremia and amicrofilaremic cases that are typically more common in children than in adults? ${ }^{7}$.

However, it is worth noting that both the AD12ICT and Og4C3-ELISA tests may yield positive results after treatment even when there is no viable infection $^{16}$, which could overestimate prevalence estimates. Conversely, because this study population was tested before MDA, it is probable that any prevalence excess due to false-positive tests would be of low magnitude. Additionally, the data draw attention to the low sensitivity of the parasitological methods, particularly the thick blood film test, in the screening of filarial infection at younger ages.

Several studies have shown that the sensitivity of both parasitological and antigen tests decreases as the MF loads decrease $\mathrm{e}^{21,31,33,41}$. This decrease may result in differential performance across population subgroups. In this study, the MF loads were slightly higher in subjects younger than 10 years old and in girls, when measured by the parasitological methods, although these differences were not statistically 
significant. Similarly, we found no significant differences in antigenemia according to age or gender. Thus, any variation in test accuracy across these subgroups of this particular population may be limited.

The prevalence estimated by Knott's test and the filtration test was, in general, slightly higher than that estimated by the thick film test. This finding implies that the former tools are most appropriate for filariasis screening of school-age populations before antifilarial treatment. Knott's technique is more laborious than the filtration technique but is usually less expensive ${ }^{42,43}$, and it showed a similar performance in detecting microfilaremia (15.3 vs. $12.0 \mathrm{MF} / \mathrm{mL})$. Thus, it may be preferred in areas with limited financial resources. A further advantage of this test is its ability to differentiate species of filaria, which makes it useful in other endemic areas where co-infection with other filarial parasites occurs.

The analysis according to age showed that the prevalence was significantly higher in the 10-15-year-old age group than in the 4-9-year-old age group, as evaluated by all parasitological methods. However, no difference in the prevalence of antigenemia between age groups according to the ICT and $\mathrm{Og} 4 \mathrm{C} 3$ tests was observed. Moreover, the prevalence of infection estimated by the antigen tests (ICT and $\mathrm{Og} 4 \mathrm{C} 3$ ) was approximately seven times higher than that estimated by the parasitological methods in the younger age group (4-9 years). In the older group (10-15 years), although the prevalence estimated by the antigen tests was again higher than that estimated by the parasitological methods, this difference was less pronounced. These results demonstrate the lower sensitivity of the parasitological methods in the younger age groups compared with the older population, as a larger proportion of individuals in the prepatent stages or who have amicrofilaremic infections (with adult worms but with no or few reproducing females) are usually found in this population, as previously described ${ }^{7,33}$.

The performance evaluation of the AD12-ICT card test and Og4C3-ELISA against the gold standard of the combination of the parasitological test results demonstrated $100 \%$ sensitivity for both tests. Additionally, the values of LR+ and LR- were compatible with the high accuracy of these tests in the diagnosis of infection in this study population, even when considering that these measures may be distorted due to the imperfection of the gold standard due to the combination of parasitological results. The LR+ for both tests may represent an underestimate because subjects with an amicrofilaremic infection could have been misclassified as free of disease according to the gold standard. Similarly, the low PPVs of the AD12-ICT card and $\mathrm{Og} 4 \mathrm{C} 3$-ELISA tests can also be explained by the gold standard, which is assumed to have a lower sensitivity ${ }^{27}$ than the antigen-based tests (AD12 and OG4C3 tests).

In regions with ongoing elimination programs, it is important to use high-specificity tests to ascertain the actual status of transmission in the population with reasonable
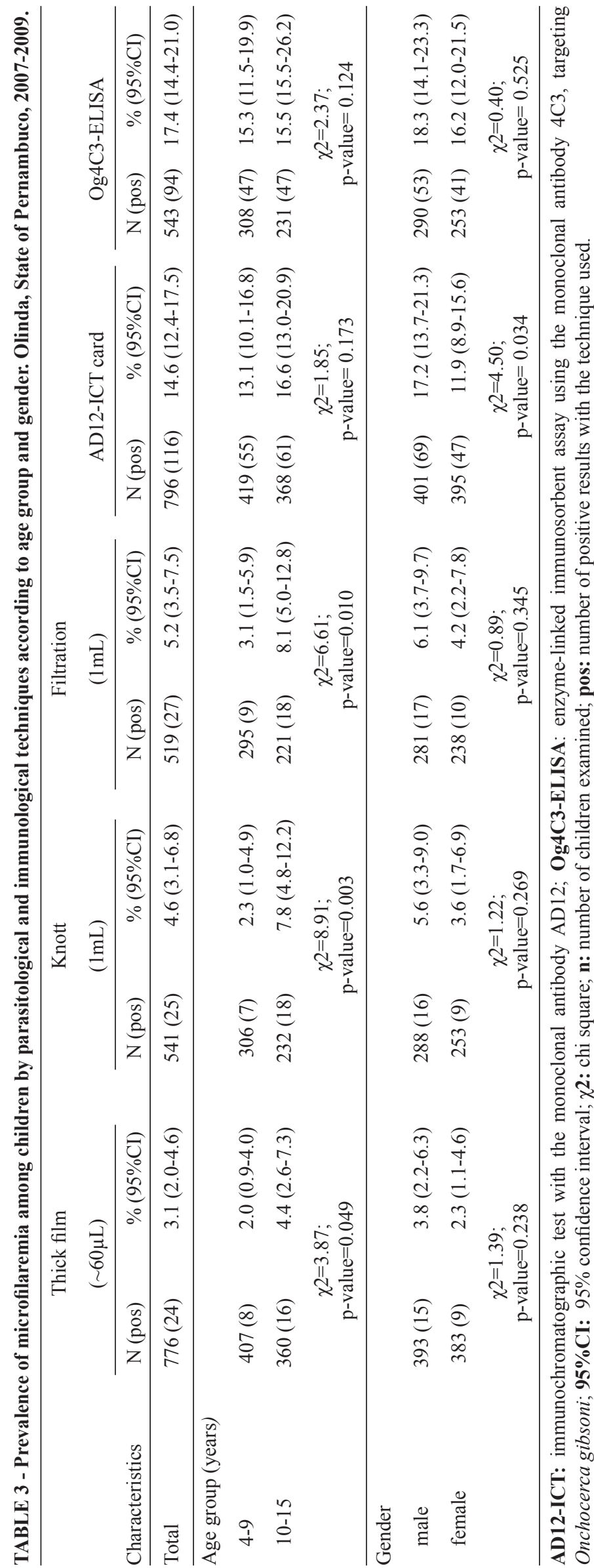
TABLE 4 - Sensitivity, positive predictive value, negative predictive value, accuracy, and likelihood ratio of the ICT card and Og4C3ELISA tests compared with those of the combination of all parasitological tests (gold standard).

\begin{tabular}{lcr}
\hline Diagnostic performance & AD12-ICT card test $(\mathrm{n}=546)$ & Og4C3-ELISA $(\mathrm{n}=542)$ \\
\hline Sensitivity \% $(95 \% \mathrm{CI})$ & $100.0(85.4-100.0)$ & $100.0(85.4-100.0)$ \\
PPV \% $(95 \% \mathrm{CI})$ & $27.8(19.8-37.7)$ & $30.8(22-41.3)$ \\
NPV \% $(95 \% \mathrm{CI})$ & $100.0(98.9-100.0)$ & $94.6(98.9-100.0)$ \\
Likelihood positive $(95 \% \mathrm{CI})$ & $6.9(5.6-8.5)$ & $7.9(6.3-9.9)$ \\
Likelihood negative & 0.0 & 0.0 \\
Accuracy \% (95\% CI) & $86.2(83.0-88.9)$ & $88.0(84.9-90.5)$
\end{tabular}

AD12-ICT: immunochromatographic test with the monoclonal antibody AD12; Og4C3-ELISA: enzyme-linked immunosorbent assay with the 4C3 monoclonal antibody, targeting Onchocerca gibsoni; 95\% CI: 95\% confidence interval; PPV: positive predictive value; NPV: negative predictive value.

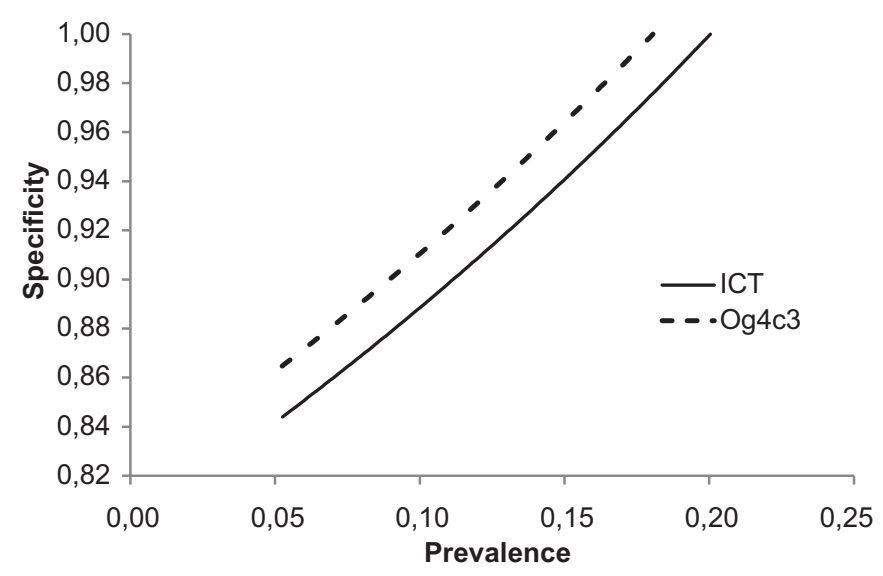

FIGURE 1 - Estimated specificity of the AD12-ICT card test and $\mathrm{Og} 4 \mathrm{C} 3$-ELISA according to the prevalence of microfilaria determined by the combination of all parasitological tests.

AD12-ICT: immunochromatographic test with the monoclonal antibody AD12; Og4C3-ELISA: enzyme-linked immunosorbent assay using the monoclonal antibody $4 \mathrm{C} 3$, targeting Onchocerca gibsoni.

certainty. Although the parasitological methods are $100 \%$ specific, they lose their usefulness after the initiation of MDA due to the potent microfilaricide effect of the antifilarial drugs, which yield a dramatic decrease in microfilaremia ${ }^{16,44}$. However, studies have shown that patients can remain infected with detectable levels of antigenemia even after several courses of treatment $t^{16,24,45,46}$. These issues limit the use of the antigen-based tests, such as the $\mathrm{AD}-12$ and $\mathrm{Og} 4 \mathrm{C} 3$ tests, in the assessment of treatment effectiveness, both at the individual and population levels.

The comparative evaluation demonstrated the high specificity of the AD12-ICT and Og4C3-ELISA tests in the study population, although a slightly higher specificity of the $\mathrm{Og} 4 \mathrm{C} 3$ test was observed when the different levels of prevalence were taken into account. Similar results were also found by Gass et al..$^{40}$, who demonstrated that the Og4C3-ELISA test showed a greater specificity. These results suggest that the $\mathrm{Og} 4 \mathrm{C} 3$ -
ELISA test is more appropriate for assessing the interruption of filarial transmission in areas with ongoing elimination programs because it yields more accurate negative results, in addition to providing quantitative results that enable the monitoring of MDA effectiveness ${ }^{18}$. Furthermore, the Og4C3-ELISA test can be performed using filter paper, which facilitates the attainment of samples in field studies as well as in venous blood samples. However, there is a need for better standardization of the technique, as well as better quality control of the kits by the manufacturer, particularly with regard to the plates and reagents.

Based on these results, we conclude that for an initial assessment of possible transmission of $W$. bancrofti, the ICT card test is the ideal tool for surveys and for mapping infection in the school-age population. The high accuracy of the AD12ICT card test, in addition to other features such as rapidity and the simplicity of execution, makes this test the method of choice for the screening of filarial infection in this population prior to the initiation of antifilarial treatment.

Due to their low sensitivity, the parasitological methods, particularly the thick film technique, are very limited in their usefulness for the estimation of the burden of infection in this population even before treatment. The ICT card test is considered the most suitable test due to its high sensitivity and, as previously mentioned, its lower specificity in populations that have already been treated ${ }^{16,46-48}$. Finally, in areas with ongoing or completed MDA, the interruption of transmission, as assessed by the tracking of incident cases of filariasis by the ICT card test in the school-age population without prior antifilarial treatment, is the preferred approach.

\section{ACKNOWLEDGMENTS}

The authors thank the National Reference Service for Filariasis (CPqAM/FIOCRUZ) in Recife and the Education and Health Departments of the City of Olinda for their support of the laboratory procedures and the field work. 


\section{CONFLICT OF INTEREST}

The authors declare that there is no conflict of interest.

\section{FINANCIAL SUPPORT}

This research was funded by the Secretaria de Vigilância em Saúde/Fundação Oswaldo Cruz/Fundação para o Desenvolvimento Tecnológico em Saúde - Vice-Presidência de Pesquisa e Laboratórios de Referência (FIOCRUZ/FIOTECVPPLR)-002-LIV11-2-1 Project.

\section{REFERENCES}

1. World Health Organization. Global Programme Eliminate Lymphatic Filariasis; progress report on mass drug administration in 2012. Wkly Epidemiol Rec 2013; 88:389-400.

2. Jaeschke R, Guyatt G, Sackett DL. Users' Guides to the Medical Literature. III How to Use an Article About a Diagnostic Test. A. Are the Results of the Study Validate. JAMA 1994; 271:389-391.

3. Reid C, Lachs MS, Feinstein AR. Use of methodological standards in diagnostic test research. JAMA 1995; 274:645-651.

4. Ramzy RMR, Hafez ON, Gad AM, Faris R, Harb M, Buck AA, et al. Efficient assessment of filariasis endemicity by screening for filarial antigenemia in a sentinel population. Trans R Soc Trop Med Hyg 1994; $88: 41-44$

5. World Health Organization. Monitoring and epidemiological assessment of the programme to eliminate lymphatic filariasis at implementation unit level. WHO/CDS/CPE/CEE/2005.50; 2005.

6. World Health Organization. Transmission assessment surveys in the Global Programme to Eliminate Lymphatic Filariasis. WHO; 2012.

7. Witt C, Ottesen EA. Lymphatic filariasis: an infection of childhood. Trop Med Int Health 2001; 6:582-606.

8. Shenoy RK. Lymphatic filariasis in children. J Commun Dis 2006; 38:118-123.

9. Shenoy RK, Suma TK, Kumaraswami V, Rahmah N, Dhananjayan G, Padma S, et al. Preliminary findings from a cross-sectional study on lymphatic filariasis in children, in an area of India endemic for Brugia malayi infection. Trop Med Parasitol 2007; 101:205-213.

10. Instituto Brasileiro de Geografia e Estatística. Censo demográfico 2010: Características populacionais e domiciliares, Rio de Janeiro, Brasil: IBGE; 2010. [Cited 2010 June 30]. Available at: http://www.ibge.gov.br/.

11. Medeiros Z, Gomes J, Beliz F, Coutinho A, Dreyer P, Dreyer G. Screening of army soldiers for Wuchereria bancrofti infection on metropolitan Recife region, Brazil. Trop Med Int Health 1999; 4:499-505.

12. Braga C, Dourado MI, Ximenes RA, Alves L, Brayner F, Rocha A, et al. Field evaluation of the whole blood immunocromatographic test for rapid bancroftian filariasis diagnosis in the northeast of Brazil. Rev Inst Med Trop Sao Paulo 2003; 45:125-129.

13. Eberhard ML, Lammie PJ. Laboratory diagnosis of filariasis. Clin Lab Med 1991; 11:977-1010.

14. Knott JA. Method for making microfilarial surveys on day blood. Trans R Soc Trop Med Hyg 1939; 32:191-196.

15. Dennis DT, Kaen BH. Isolation of microfilariae: report of new method. J Parasit 1971; 57:1146-1147.

16. Weil GJ, Ramzy RMR. Diagnostic tools for filariasis elimination programmes. Trends Parasitol 2007; 23:78-82.

17. More SJ, Copeman DB. A highly specific and sensitive monoclonal antibody-based ELISA for the detection of circulating antigen in bancroftian filariasis. Trop Med Parasitol 1990; 41:403-406.
18. Lalitha P, Ravichandra M, Suba S, Kaliraj P, Narayanan RB, Jayaraman K Quantitative assessement of circulating antigens in human lymphatic filariasis: a field evaluation of monoclonal antibody-based ELISA using blood collected on filter strips. Trop Med Int Health 1998; 3:41-45.

19. Weil GJ, Jain DC, Santhanam S, Malhotra A, Kumar H, Sethumadhavan KVP, et al. A monoclonal antibody-based enzyme immunoassay for detecting parasite antigenemia in bancroftian filariasis. J Infec Dis 1987; 156:350355.

20. Eberhard ML, Hightower AW, Addiss DG, Lammie PJ. Clearance of Wuchereria bancrofti antigen after treatment with diethylcarbamazine or ivermectin. Am J Trop Med Hyg 1997; 57:483-486.

21. Nicolas L, Plichart C, Nguyen LN, Moulia-Pelat JP. Reduction of Wuchereria bancrofti adult worm circulating antigen after annual treatments of diethylcarbamazine combined with ivermectin in French Polynesia. J Infec Dis 1997; 175:489-492.

22. Ismail MM, Weil GJ, Jayasinghe KSA, Premaratne UN, Abeyewickreme W, Rajaratnam HN, et al. Prolonged clearance of microfilaraemia in patients with bancroftian filariasis after multiple high doses of ivermectin or diethylcarbamazine. Trans R Soc Trop Med Hyg 1996; 90:684-688.

23. Weil GJ, Lammie PJ, Weiss N. The ICT filariasis test: A rapid-format antigen test for diagnosis of bancroftian filariasis. Parasitol Today 1997; $13: 401-404$

24. Freedman DO, Plier DA, Almeida AB, Oliveira AL, Miranda J, Braga C. Effect of aggressive prolonged diethylcarbamazine therapy on circulating antigen levels in bancroftian filariasis. Trop Med Int Health 2001; 6: $37-41$.

25. Wilson K, Grenfell BT. Generalized linear modelling for parasitologists. Parasitol Today 1997; 13:33-38.

26. Deeks JJ, Altman DG. Statistics Notes: Diagnostic tests 4: likelihood ratios. BMJ 1994; 329:168.

27. Staquet M, Rozencweig M, Lee YJ, Muggia FM. Methodology for the assessment of new dichotomous diagnostic tests. J Chronic Dis 1981; 34:599-610.

28. Hayen A, Macaskill P, Irwig L, Bossuyt P. Appropriate statistical methods are required to assess diagnostic tests for replacement, add-on, and triage. J Clin Epidemiol 2010; 63:883-991.

29. Chanteau S, Moulia-Pelat JP, Glaziou P, Nguyen NL, Luquiaud P, Plichart C, et al. $\mathrm{Og} 4 \mathrm{C} 3$ circulating antigen: a marker of infection and adult worm burden in Wuchereria bancrofti filariasis. J Infect Dis 1994; 170:247-250.

30. Lammie PJ, Hightower AW, Eberhard ML. The age-specific prevalence of antigenemia in a Wuchereria bancrofti-exposed population. Am J Trop Med Hyg 1994; 51:348-355.

31. Rocha A, Addiss D, Ribeiro ME, Noroes J, Baliza M, Medeiros Z, et al. Evaluation of the $\mathrm{Og} 4 \mathrm{C} 3 \mathrm{ELISA}$ in Wuchereria bancrofti infection: infected persons with undetectable or ultra-low microfilarial densities. Trop Med Int Health 1996; 1:859-864.

32. Freedman DO, Almeida A, Miranda J, Plier DA, Braga C. Field trial of a rapid card test for Wuchereria bancrofti. Lancet 1997; 350:1681.

33. Itoh M, Weerasooriya MV, Gunawardena NK, Mudalige MPS, Samarawickrema WA, Kimura E. Wuchereria bancrofti antigenemia in Sri Lanka. Trop Med Int Health 1999; 4:207-210.

34. Ramzy RM, Helmy H, el-Lethy AS, Kandil AM, Ahmed ES, Weil GJ, et al. Field evaluation of a rapid-format kit for the diagnosis of bancroftian filariasis in Egypt. East Mediterr Health J 1999; 5:880-887.

35. Omar MS, Sheikha AK, Al-Amari OM, Abdalla SE, Musa RA. Field evaluation of two diagnostic antigen tests for Wuchereria bancrofti infection among Indian expatriates in Saudi Arabia. Southeast Asian J Trop Med Public Health 2000; 31:415-418.

36. Njenga SM, Wamae CN. Evaluation of ICT filariasis card test using whole capillary blood: comparison with Knott's concentration and counting chamber methods. J Parasitol 2001; 87:1140-1143.

37. Chandrasena TG, Prematura R, Abeyewickrema W, Silva NR. Evaluation of the ICT whole-blood antigen card test to detect infection due to Wuchereria bancrofti in Sri Lanka. Trans R Soc Trop Med Hyg 2002; 96:60-63. 
38. Bal MS, Beuria MK, Mandal NN, Das MK. Antigenemia in young children living in Wuchereria bancrofti endemic areas of Orissa, India. Trans R Soc Trop Med Hyg 2009; 103:262-265.

39. Rocha A, Braga C, Belém M, Carrera A, Aguiar-Santos AM, Oliveira P, et al. Comparison of tests for the detection of circulating filarial antigen $(\mathrm{Og} 4 \mathrm{C} 3-$ ELISA and AD12-ICT) and ultrasound in diagnosis of lymphatic filariasis in individuals with microfilariae. Mem Inst Oswaldo Cruz 2009; 104:621-625.

40. Gass K, Rochars MVB, Boakye D, Bradley M, Fischer PU, Gyapong J, et al. A multicenter evaluation of diagnostic tools to define endpoints for programs to eliminate bancroftian filariasis. PLoS Negl Trop Dis 2012, 6:e1479.

41. Rocha A, Lima G, Medeiros Z, Aguiar-Santos A, Alves S, Montarroyos U, et al. Circulating filarial antigen in the hydrocele fluid from individuals living in a bancroftian filariasis area-Recife, Brazil: detected by the monoclonal antibody Og4C3-assay. Mem Inst Oswaldo Cruz 2004; 99:101-105.

42. Rocha A. Métodos laboratoriais disponíveis para o diagnóstico da filariose linfática. RBAC 2000; 32:265-270.

43. Melrose WD, Turner PF, Pisters P, Turner B. An improved Knott's concentration test for the detection of microfilariae. Trans R Soc Trop Med Hyg 2000; 94:176.
44. Ottesen EA, Hooper PJ, Bradley M, Biswas G. The Global Programme to Eliminate Lymphatic Filariasis: Health Impact after 8 Years. PLOS Negl Trop Dis 2008; 2:1-12.

45. Schuetz A, Addiss DG, Eberhard ML, Lammie PJ. Evaluation of the whole blood filariasis ICT test for short-term monitoring after antifilarial treatment. Am J Trop Med Hyg 2000; 62:502-503.

46. Ramzy RM, El Setouhy M, Helmy H, Ahmed ES, Abd Elaziz KM, Farid HA, et al. Effect of yearly mass drug administration with diethylcarbamazine and albendazole on bancroftian filariasis in Egypt: a comprehensive assessment. Lancet 2006; 367:992-999.

47. Njenga SM, Wamae CN, Njomo DW, Mwandawiro CS, Molyneux DH. Impact of two rounds of mass treatment with diethylcarbamazine plus albendazole on Wuchereria bancrofti infection and the sensitivity of immunochromatographic test in Malindi, Kenya. Trans R Soc Trop Med Hyg 2008; 102:1017-1024.

48. Tisch DJ, Bockarie MJ, Dimber Z, Kiniboro B, Tarongka N, Hazlett FE, et al. Mass drug administration trial to eliminate lymphatic filariasis in Papua New Guinea: changes in microfilaremia, filarial antigen, and Bm14 antibody after cessation. Am J Trop Med Hyg 2008; 78:289. 